UB-ECM-PF-92/15

\title{
Anomalies in the effective theory of heavy quarks*
}

\author{
Joan Soto $\nmid$ and \\ Rodanthy Tzani $\ddagger$ \\ Departament d'Estructura i Constituents de la Matèria, \\ Universitat de Barcelona, Barcelona, Catalonia, Spain
}

\begin{abstract}
The question of the anomalies in the effective theory of heavy quarks is investigated at two different levels. Firstly, it is shown that none of the symmetries of this effective theory contains an anomaly. The existence of a new ' $\gamma_{5}$ '-symmetry is pointed out and shown to be also anomaly free. Secondly, it is shown that the chiral anomaly of QCD is not reproduced in the effective lagrangian for the heavy quarks, thus contradicting 't Hooft's anomaly matching condition. Finally, the effective theory of heavy quarks is derived from the QCD lagrangian in such a way that the terms leading to the anomaly are included. For this derivation the generating functional method is used.
\end{abstract}

\footnotetext{
* This research was supported in part by the CICYT grant AEN90-0033. R.T. also acknowledges a grant from the Ministerio de Educación y Ciencia, Spain

$\dagger$ soto@ebubecm1.bitnet

$\ddagger$ tzani@ebubecm1.bitnet
} 


\section{Introduction}

The heavy quark effective theory (HQET), introduced originally by Isgur and Wise [1], is a theory in which all the dependence on the large rest mass of the heavy quark has been removed analytically. In particular, one assumes the heavy quark non-relativistic (essentially static) and the theory is an expansion in the heavy quark spacial momentum over its rest energy, that is an $\frac{1}{m}$ expansion [2].

The success of the formalism is based on the fact that the lowest order in the expansion exhibits a number of new symmetries that are not present in the original QCD lagrangian. One therefore can obtain model independent results using only the symmetries of the theory in order to relate matrix elements of physical processes $[2,3]$.

There exist essentially two different approaches for the lagrangian formulation of this effective theory. The first one, which is conceptually the most direct, is based on the non-relativistic approximation of the Dirac theory. Performing directly a non-relativistic approximation in Dirac theory one obtains the non-relativistic Schrödinger-Pauli theory for a two component field, which describes either quarks or antiquarks, as an expansion in $\frac{1}{m}[4]$.

The second formalism, due to Georgi [5], makes use of a new field $h$ which contains only small fluctuations of the momentum. In other words, the original quark field, redefined as

$$
\Psi=e^{-i m \psi v_{\mu} x^{\mu}} h_{v}(x)=\left(\frac{1+\psi}{2}\right) e^{-i m v \cdot x} h_{v}^{+}(x)+\left(\frac{1-\psi}{2}\right) e^{i m v \cdot x} h_{v}^{-}(x)
$$

is almost on shell, except for small momenta fluctuations described by the field $h_{v}=$ $h_{v}^{+}+h_{v}^{-}$. The 4 -velocity of the system, $v^{\mu}$, is essentially the quark velocity in the $m \rightarrow \infty$ limit $\left(v_{\mu} v^{\mu}=1, v_{0}<0\right)$. One then can show that the original QCD lagrangian

$$
i \bar{\Psi} \not D \Psi-m \bar{\Psi} \Psi
$$

( $D_{\mu}=\partial_{\mu}+B_{\mu}, B_{\mu}$ being the $s u(3)$-valued gluon field) can be approximated by an effective theory in terms of the field $h_{v}$, in which the mass dependence has been removed from the Dirac operator. Namely,

$$
L_{e f f}=i \bar{h}_{v} \psi v_{\mu} D^{\mu} h_{v}=i \bar{h}_{v}^{+} \not \supset h_{v}^{+}+i \bar{h}_{v}^{-} \not D h_{v}^{-}
$$

where in the last expression $h_{v}^{+}$and $h_{v}^{-}$denote the quark annihilation operator and antiquark creation operator correspondingly and obey the relation $\psi h_{v}^{ \pm}= \pm h_{v}^{ \pm}$. The subindex 
$v$ denotes the dependence of the quark fields on the velocity. The basic idea of Georgi's effective theory is to keep explicit the Lorentz covariance and hence exhibit all the symmetries of the theory. This is, however, the lowest order in the $\frac{1}{m}$ expansion and is not obvious how one should proceed in order to include higher order corrections in the action.

In a later paper Mannel et al. [6] have derived the effective lagrangian in the first order in $\frac{1}{m}$ using the functional integral approach. The advantage of this last formalism is that in the rest frame it can be shown to be equivalent with the non-relativistic approximation of ref. [4], keeping, therefore, the physics more transparent. In their formalism, however, it seems crucial to keep in the theory only one kind of fields either quarks or antiquarks, but not both.

In this work we shall restrict ourselves to purely theoretical aspects of the heavy quark theory (see ref. [3] for a review on phenomenological applications) which have not been discussed so far. In particular, we shall be concerned with anomalies. We are going to address the issue at two distinct levels. In the first one, we consider Georgi's effective action (3) and answer the question whether the flavor, spin, and an extra symmetry, which we shall point out, are anomalous in the negative. This confirms that the symmetries of the effective theory are actually symmetries of the full quantum theory so that they can be safely used to relate form factors as it has been done so far. The second level addresses the question on how the chiral anomaly of the fundamental theory is realized in the effective theory.

This is a low energy effective theory and according to 't Hooft consistency condition one expects the same anomaly as in the original one. On the other hand, since in this approximation the heavy quark and antiquark fields are separated by the infinite mass gap, the original axial U(1) symmetry is lost. How then this low energy effective theory realizes the chiral anomaly of the fundamental high energy theory? In order to answer this question we set a formalism based on the functional integral method. In this way, the picture of the chiral anomaly becomes transparent. We are also able to calculate the $\frac{1}{m}$ corrections of Georgi's lagrangian in a conceptually simple and systematic way (i.e. we avoid having to integrate out the antiparticles as in ref. [6]). Moreover, in our formalism it becomes clear that one can describe on the same footing any number of flavors, quarks or antiquarks, with equal or different velocities. 


\section{Anomalies in the symmetries of the HQET}

In order to discuss the question of anomalies, we first analyze the symmetries in Georgi's effective action (3). In what follows we use the notation of ref. [5]. There exist the following symmetries in this theory:

$$
h_{v} \rightarrow e^{i \theta} h_{v} \text { and } h_{v} \rightarrow e^{i \psi \theta^{\prime}} h_{v}
$$

with Noether's currents

$$
j^{\mu}=\bar{h}_{v} \psi v^{\mu} h_{v} \text { and } j^{\mu^{\prime}}=\bar{h}_{v} v^{\mu} h_{v}
$$

correspondingly. These symmetries correspond to changing the field components $h_{v}^{+}$and $h_{v}^{-}$by arbitrary phases and result to the conservation of the quark and antiquark number currents independently. (4) becomes the flavor symmetry

$$
\left(h_{v}^{ \pm}\right)^{j} \rightarrow\left(e^{i \theta_{ \pm}}\right)_{i}^{j}\left(h_{v}^{ \pm}\right)^{i}
$$

in a many flavor lagrangian. Where now $\theta_{ \pm}$is an arbitrary traceless hermitian matrix in the flavor space and in this case the currents (5) are matrices in flavor space. There exist also the so called 'spin' symmetry in this lagrangian, as it is discussed in ref. [1] and [5]. In particular, the action (3) is invariant under the transformation

$$
h_{v}^{ \pm} \rightarrow e^{i \epsilon_{ \pm}^{i} S_{i}^{ \pm}} h_{v}^{ \pm} \text {and } \bar{h}_{v}^{ \pm} \rightarrow \bar{h}_{v}^{ \pm} e^{-i \epsilon_{ \pm}^{i} S_{i}^{ \pm}}
$$

where $S_{i}^{ \pm}=i \epsilon_{i j k}\left[\phi_{j}, \phi_{k}\right](1 \pm \psi) / 2$ and the parameters of the transformation $\epsilon_{ \pm}^{i}$ are three arbitrary hermitian matrices in the flavor space. The orthonormal set of the space like vectors $e_{j}^{\mu}, j=1,2,3$ are orthogonal to $v^{\mu}$. For one flavor, the classically conserved current due to the spin symmetry is

$$
j_{i}^{\mu, \pm}=\bar{h}_{v}^{ \pm} \psi v^{\mu} S_{i}^{ \pm} h_{v}^{ \pm} .
$$

For many flavors, a matrix in flavor space must be inserted in the current (8). Notice that for the flavor and spin symmetries the fact that we have both quark and antiquark fields in our theory is not essential. The above symmetries would persist even if we dropped either field.

It is not easy to keep track of the axial symmetry of the original theory (2) in this approximation. The invariance of (2) under the axial transformation manifests itself only 
in the limit $m \rightarrow 0$. It is broken explicitly by the mass term and in the limit $m \rightarrow \infty$, which is the limit of the effective theory, it can not be realized in a simple way.

There exists, however, an extra symmetry in the HQET which formally resembles the axial symmetry of the original theory being nevertheless unrelated to it. Classically the action of the HQET is invariant under the transformation

$$
h_{v} \rightarrow e^{i \gamma_{5} \epsilon} h_{v} \text { and } \bar{h}_{v} \rightarrow \bar{h}_{v} e^{i \gamma_{5} \epsilon}
$$

where again $\epsilon$ is a matrix in the flavor space. It is worth noting that this symmetry is present only when one insists in having the quark and antiquark fields with the same velocity. For the rest of the discussion we shall refer to this symmetry as ' $\gamma_{5}$ ' in order to distinguish it from the axial symmetry of the original theory. Under this ' $\gamma_{5}$ '-symmetry the field components transform as follows

$$
h_{v}^{ \pm} \rightarrow \cos \epsilon h_{v}^{ \pm}+i \gamma_{5} \sin \epsilon h_{v}^{\mp} .
$$

This is an unexpected symmetry since it mixes quark and antiquark fields, while in the lagrangian of the HQET the terms which mix quarks and antiquarks are neglected. This is a low energy effective theory which can not describe heavy quark pair production, since it would require very large momentum transfer. The physical relevance of this last symmetry is not very clear. The conserved quantity corresponding to this symmetry has no obvious physical interpretation. One can show that the original axial symmetry coincides with the last $\gamma_{5}$-symmetry in the limit $m \rightarrow 0$. Indeed, under the axial transformation on the field $\Psi$, the redefined field components transform as follows

$$
h_{v}^{ \pm} \rightarrow \cos \epsilon h_{v}^{ \pm}+i \gamma_{5} \sin \epsilon e^{ \pm 2 i m v \cdot x} h_{v}^{\mp} .
$$

When $m=0$ (11) coincides with (10), but whenever $m \neq 0$ the two transformations become independent. Since, however, (1) is not a good field redefinition in the limit $m \rightarrow 0$, in the sense that the lagrangian of the HQET (3) cannot be derived in this limit, this coincidence can be superficial.

It is, now, easy to show that neither the spin nor the flavor symmetries are anomalous in this theory. In both cases for the computation of the divergence of the current, one has to compute the trace of the commutator between the symmetry operator and the inverse propagator $\boldsymbol{A}$. Indeed for the spin symmetry the relevant quantity is

$$
\operatorname{tr}\left(\left[\epsilon_{ \pm}^{i} S_{i}^{ \pm}, \Delta\right] \frac{1}{\not} G_{r e g}\right)
$$


where $\not \alpha=\gamma^{\mu} \Delta_{\mu}$ with $\Delta_{\mu}:=v_{\mu} v \cdot D$. For the flavor symmetry it is enough to compute

$$
\left.\operatorname{tr}([\theta, \not]] \frac{1}{\not \alpha} G_{r e g}\right) \text { and } \operatorname{tr}\left(\left[\psi \theta^{\prime}, \not x\right] \frac{1}{\not} G_{r e g}\right)
$$

where $\epsilon_{ \pm}^{i}, \theta$ and $\theta^{\prime}$ are matrices in flavor space and $G_{r e g}$ denotes the regulator. Choosing then the regulator in such a way that it preserve the vector symmetry, namely to be an appropriate function of $\boldsymbol{X}$, and using trace properties the above commutators are zero.

For the case of the ' $\gamma_{5}$ '-symmetry one needs to compute the analogous anticommutator, in order to calculate the divergence of the corresponding Noether's current

$$
j_{5}^{\mu}=\bar{h}_{v} \psi v^{\mu} \gamma_{5} h_{v} .
$$

(A matrix in flavor space must be inserted in (14) for the case of many flavors). In particular, the quantity to be computed is given by

$$
\operatorname{tr}\left(\left\{\epsilon(x) \gamma_{5}, \Delta\right\} \frac{1}{\not} G_{r e g}\right)=\operatorname{tr}\left(2 \epsilon(x) \gamma_{5} G_{r e g}\right)
$$

where again the regulator $G_{r e g}$ is an appropriate function of $\Delta=\not v_{\mu} D^{\mu}$. Then, using trace properties, the fact that $\psi^{2}=1$ and the property $\psi \gamma_{5}=-\gamma_{5} \psi$, it is easy to show that this gives zero. Indeed,

$$
\begin{aligned}
\operatorname{tr}\left(2 \epsilon(x) \gamma_{5} G_{r e g}\right) & =\operatorname{tr}\left(2 \epsilon(x) \psi^{2} \gamma_{5} G_{r e g}\right)=\operatorname{tr}\left(2 \epsilon(x) \psi \gamma_{5} G_{r e g} \psi\right) \\
& =-\operatorname{tr}\left(2 \epsilon(x) \psi^{2} \gamma_{5} G_{r e g}\right)=0 .
\end{aligned}
$$

With this we conclude that none of the symmetries in Georgi's action is anomalous.

The physical reasoning behind the cancellation of the ' $\gamma_{5}$ '- anomaly, is very interesting and it can be understood in many different ways. In obtaining (3) from (2) one neglects in the lagrangian terms that involve quark-antiquark pairs. Therefore, since pair production is not relevant in this approximation, the triangle graph is not possible in this effective theory and hence there is no anomaly.

The phenomenon that the divergence of the $j_{5}^{\mu}$ current is zero in this effective theory is related to taking the large mass limit in the fermionic massive theory (2). When $m$ becomes large, the mass term in the action (2) plays the role of Pauli-Villars regulator with fields of opposite statistics. Therefore in the limit $m \rightarrow \infty$ the contribution from the mass term 
in the divergence of the axial current is equal in magnitude and opposite in sign with the contribution from the anomaly. The two contributions, therefore, cancel each other and the divergence of the current is zero in this limit. This could be a satisfactory explanation if the axial current of (2) coincided with the $j_{5}^{\mu}$ current of the effective theory. In this case, however, the original axial current can not be related to the $j_{5}^{\mu}$ in any straightforward way. On the other hand, the effective theory of heavy quarks is an expansion in $\frac{1}{m}$ and in principle the matrix elements of physical processes could receive contributions from the divergence of the current in some higher order in $\frac{1}{m}$. The action (3) is only a leading order in the expansion and hence the dominant $\frac{1}{m}$ contributions in the divergence of the current are missed in this approximation. The question of the chiral anomaly, therefore, in this heavy quark effective theory needs more investigation. In what follows we analyze the chiral anomaly of the fundamental theory in the heavy quark approximation.

\section{The chiral anomaly in the HQET}

Once we have seen that none of the symmetries of the HQET is anomalous, we now discuss the realization of the $U_{L}\left(N_{f}\right) \otimes U_{R}\left(N_{f}\right)$ symmetry of the classical QCD lagrangian with $N_{f}$ massless flavors in this effective theory. This symmetry is explicitly broken both by the quark masses and by the regularization. The latter breaking leads to the chiral anomaly. Even though the explicit breaking due to the quark masses is very large for heavy quarks the question above is still relevant. The chiral anomaly does not depend on the quark masses and breaks the $U_{L}\left(N_{f}\right) \otimes U_{R}\left(N_{f}\right)$ symmetry down to the vector subgroup $U_{V}\left(N_{f}\right)$. (We understand that suitable sources are added to the QCD lagrangian such that the above symmetries become local, otherwise only the $U_{L}(1) \otimes U_{R}(1)$ part is broken down to $U_{V}(1)$ by the anomaly whereas $S U_{L}\left(N_{f}\right) \otimes S U_{R}\left(N_{f}\right)$ is spontaneously broken by non-perturbative effects down to $S U_{V}\left(N_{f}\right)$ ). Furthermore, the chiral anomaly is given by one loop diagrams only and the anomalous current conservation equation is not renormalized at any order in perturbation theory [7]. These peculiar features together with some important physical inputs related to the inconsistency of chiral gauge theories [8] motivated the so-called 't Hooft anomaly matching condition [9] which in a wide sense (see [10] for more precise discussions) can be stated as follows: any low-energy effective theory must reproduce the chiral anomaly of the fundamental theory from which it is a low energy realization. The 't Hooft anomaly matching condition, for instance, requires 
the introduction of a Wess-Zumino term in the low energy realization of QCD with $N_{f}$ $\left(N_{f}>2\right)$ flavors by chiral lagrangians [11]. The HQEL can be regarded as a low energy realization of QCD for $m_{Q}>>\Lambda_{Q C D}$. Adopting, then, the point of view that 't Hooft anomaly matching conditions should be fulfilled even for large mass theories, we investigate the question of chiral anomaly in the HQEL.

Since $m_{Q}$ is large the explicit breaking of $U_{L}\left(N_{f}\right) \otimes U_{R}\left(N_{f}\right)$ symmetry is also large in HQET. In order to separate the explicit breaking due to $m_{Q}$ from the breaking due to the regularization it is convenient to enlarge our original theory by adding sources with appropriate transformation properties under the gauge group in such a way that the action is explicitly invariant [11]. Consider, then, our original lagrangian in Euclidean space including the sources to be

$$
L=\bar{\Psi}\left(\gamma^{\mu} D_{\mu}+\Phi\right) \Psi
$$

where $D_{\mu}=\partial_{\mu}+A_{\mu} \quad$ and $\quad A_{\mu}:=A_{\mu}^{L} P_{L}+A_{\mu}^{R} P_{R}$ is a $U_{L}\left(N_{f}\right) \otimes U_{R}\left(N_{f}\right)$ gauge field (i. e. $A_{\mu}=A_{\mu}^{a} T_{a}$ with $T_{a}$ being the $U\left(N_{f}\right)$ generators). $P_{L, R}$ denote the projection operators, $P_{L, R}=\frac{1 \pm \gamma_{5}}{2}$. In writing (17) we have promoted the mass term into a scalar field source $\Phi$ defined by

$$
\Phi:=\phi P_{L}-\phi^{\dagger} P_{R}
$$

Under the local chiral transformation, the fields $\Psi, \bar{\Psi}$ and $A_{\mu}$ transform as

$$
\Psi \longrightarrow g \Psi, \quad \bar{\Psi} \longrightarrow \bar{\Psi} \hat{g}^{\dagger}, \quad A_{\mu} \longrightarrow g A_{\mu} g^{\dagger}+g \partial_{\mu} g^{\dagger},
$$

where $g$ is defined by $g:=g_{L} P_{L}+g_{R} P_{R} \quad$ with $\quad g_{L, R} \in U\left(N_{f}\right)$ and the meaning of hats is interchanging $P_{L} \leftrightarrow P_{R}$.

Then, the action (17) is invariant under (18) if we allow $\Phi$ to transform as

$$
\Phi \longrightarrow \hat{g} \Phi g^{\dagger}
$$

Our task is now to generalize (3) in such a way that (i) the chiral symmetry (18) and (19) is preserved and (ii) (3) is recovered upon switching off the sources, that is after setting $A_{\mu}^{L}=A_{\mu}^{R}=B_{\mu}$ and $\Phi=i M$. Since $\Phi^{\dagger}=-\hat{\Phi}$ we can write

$$
\Phi=i \hat{U} M U^{\dagger} \quad \text { with } \quad U^{\dagger} U=1,
$$


where $M$ is a hermitian diagonal matrix and $U$ transforms as

$$
U \longrightarrow g U
$$

Next, we define

$$
P_{ \pm}:=\frac{1}{2}\left(1 \mp U \psi U^{\dagger}\right)
$$

where $v_{\mu}$ is a diagonal matrix in the flavor space containing the heavy quark velocities. It is easy to see that $P_{ \pm}$is a projection operator, that is

$$
P_{ \pm}^{2}=P_{ \pm} \quad, \quad P_{+} P_{-}=P_{-} P_{+}=0 \quad, \quad \hat{P}_{ \pm} \Phi=\Phi P_{ \pm} .
$$

Then, the field redefinition (1) is generalized to

$$
h_{v}^{ \pm}:=P_{ \pm} U e^{\mp i v \cdot x M} U^{\dagger} \Psi \quad \text { and } \quad \bar{h}_{v}^{ \pm}:=\bar{\Psi} \hat{P}_{ \pm} \hat{U} e^{ \pm i v \cdot x M} \hat{U}^{\dagger}
$$

and hence under the symmetry transformation (18) the quark fields transform as

$$
h_{v}^{ \pm} \longrightarrow g h_{v}^{ \pm} \text {and } \bar{h}_{v}^{ \pm} \longrightarrow \bar{h}_{v}^{ \pm} \hat{g}^{\dagger} \text {. }
$$

Substituting, now, (24) into (17) we obtain

$$
L=\bar{h}_{v}^{ \pm} D_{v}^{ \pm} h_{v}^{ \pm}
$$

where

$$
\begin{aligned}
D_{v}^{ \pm} & :=\hat{P}_{ \pm}\left[ \pm \hat{U} v^{\mu} U^{\dagger}\left(\partial_{\mu}+U \partial_{\mu} U^{\dagger}\right) \pm \hat{U} v^{\mu} e^{\mp i v \cdot x M} U^{\dagger} D_{\mu} U e^{ \pm i v \cdot x M} U^{\dagger}\right. \\
& \left.+\gamma^{\mu} P_{\mp} U e^{\mp i v \cdot x M} U^{\dagger} D_{\mu} U e^{ \pm i v \cdot x M} U^{\dagger}\right] P_{ \pm} .
\end{aligned}
$$

In obtaining the last expression we have used the relations

$$
\begin{aligned}
& \hat{P}_{ \pm} \gamma^{\mu} P_{ \pm}= \pm \hat{U} v^{\mu} U^{\dagger} P_{ \pm}= \pm \hat{P}_{ \pm} \hat{U} v^{\mu} U^{\dagger} \\
& \partial_{\mu}\left(U \psi U^{\dagger}\right) P_{ \pm}=P_{\mp} \partial_{\mu}\left(U \psi U^{\dagger}\right) .
\end{aligned}
$$

The expression (26) with the definition (27) is a generalization of (3) and gives the effective lagrangian for heavy quarks in a language in which the chiral gauge invariance is explicit. Indeed, the action is manifestly gauge invariant, since $U \partial_{\mu} U^{\dagger}$ transforms as the gauge field $A_{\mu}$. Notice also that (26) reduces to (3) upon setting $U=1$ and $A_{\mu}=B_{\mu}$.

A key observation, now, is the following: Even though $D_{v}^{ \pm}$transforms as the operator in the original (anomalous) lagrangian (17), that is

$$
D_{v}^{ \pm} \longrightarrow \hat{g} D_{v}^{ \pm} g^{\dagger}
$$


(26) can be regulated in a gauge invariant way due to the fact that now we have velocities at our disposal. Indeed, due to the transformation property (29) the eigenvalues of $D_{v}^{ \pm}$ are not chiral gauge invariant and, therefore, appears that the determinant of the operator cannot be regulated in a chiral invariant way [12]. On the other hand, since $\psi^{2}=1$, the following formal identities hold

$$
\operatorname{det}\left(D_{v}^{ \pm}\right)=1 \cdot \operatorname{det}\left(D_{v}^{ \pm}\right)=\operatorname{det}\left(\psi^{\prime}\right) \cdot \operatorname{det}\left(D_{v}^{ \pm}\right)=\operatorname{det}\left(\psi^{\prime} D_{v}^{ \pm}\right)
$$

where $\psi^{\prime}$ is a matrix proportional to the identity in flavour space made out of one of the velocities in $\not$. Now, under the transformation (18)

$$
\psi^{\prime} D_{v}^{ \pm} \longrightarrow g \psi^{\prime} D_{v}^{ \pm} g^{\dagger} .
$$

The last transformation property assures the chiral invariance of the eigenvalues of the

operator $\psi^{\prime} D_{v}^{ \pm}[12]$. Therefore, with an appropriate choice of regulator, $\operatorname{det}\left(\psi^{\prime} D_{v}^{ \pm}\right)$, and hence $\operatorname{det}\left(D_{v}^{ \pm}\right)$because of (30), can be defined in a chiral invariant way.

The conclusion of the above analysis is that the lagrangian (26) of the HQET does not reproduce the chiral anomaly of the fundamental theory. This is related to the fact that the HQET is a non-relativistic approximation (even if one writes it in a covariant form) and it is crucial to insist in full relativistic covariance in order to have chiral anomalies. If we believe in 't Hooft anomaly matching condition, then, the lagrangian (26) of the HQET must be modified in a suitable way such that the chiral anomaly is reproduced. We devote the rest of the paper to this objective.

\section{A derivation of the HQET}

In this part we present an alternative derivation of the effective theory of heavy quarks in such a way that we keep track of the chiral anomaly of the fundamental high energy theory. For this purpose we use the generating functional formalism. Consider the generating functional of heavy quark Green functions with insertions of heavy quark bilinear composite operators given by

$$
\begin{gathered}
Z\left(\bar{\eta}, \eta ; A_{\Gamma}\right)=\int D \bar{\Psi} D \Psi \exp \left[i \int_{x}(\bar{\Psi} \mathcal{D} \Psi+\bar{\eta} \Psi+\bar{\Psi} \eta)\right] \\
=\operatorname{det}(\mathcal{D}) e^{-i \int_{x} \int_{y} \bar{\eta}(x)\left(\mathcal{D}^{-1}\right)_{(x, y)} \eta(y)} \equiv e^{W}
\end{gathered}
$$


where we write explicitly only the piece of the QCD lagrangian that contains heavy quark fields. Further integrations over the gluon fields and the light quark fields with the rest of the QCD lagrangian must be understood in (32) and in the formulas derived from it. The operator $\mathcal{D}$ is given by

$$
\mathcal{D}=i \not D \cdot 1+\Gamma A_{\Gamma}-M
$$

where $\not D=\gamma^{\mu}\left(\partial_{\mu}+B_{\mu}\right)$, with $B_{\mu}$ being the gluon field and $\Gamma$ stands for any combination of Dirac matrices. For definiteness let us consider 2-flavor space (i.e., b and c quarks only), though the analysis extends trivially to $N_{f}$ flavors. The matrices $A_{\Gamma}$ and $M$ are given, then, by

$$
A_{\Gamma}=\left(\begin{array}{cc}
A_{\Gamma}^{c c} & A_{\Gamma}^{c b} \\
A_{\Gamma}^{b c} & A_{\Gamma}^{b b}
\end{array}\right) \quad \text { and } \quad M=\left(\begin{array}{cc}
m_{c} & 0 \\
0 & m_{b}
\end{array}\right)
$$

$A_{\Gamma}$ can be either scalar, axial, vector or axial vector source depending on the choice of $\Gamma$. $m_{b}, m_{c}$ denote the quark masses and $\bar{\Psi}, \Psi$ and $\bar{\eta}, \eta$ are row and column matrices correspondingly in the space of flavors.

In order to describe heavy quarks almost on shell we choose the following sources:

$$
\bar{\eta}(x)=\bar{\eta}_{v}^{+}(x) \frac{1+\psi}{2} e^{i M v \cdot x} \text { and } \eta(x)=e^{-i M v \cdot x} \frac{1+\psi}{2} \eta_{v}^{+}(x),
$$

where $\eta_{v}^{+}$and $\bar{\eta}_{v}^{+}$are slowly varying functions such that upon functional variation will generate Green functions of heavy quarks with small momentum fluctuations about $M \cdot v_{\mu}$. $v_{\mu}$ is the following matrix of the quark velocities

$$
v_{\mu}=\left(\begin{array}{cc}
v_{\mu}^{c} & 0 \\
0 & v_{\mu}^{b}
\end{array}\right)
$$

where again $v^{2}=1$. Note that with the choice of sources (35) we have chosen to describe only quark fields in our theory. Extra antiquark or quark fields with different velocities can be described by adding appropriate terms in our expressions (35).

Next, we compute the generating functional $W$ except for the determinant. The part of the generating functional to be computed is

$$
\int_{x} \int_{y} \bar{\eta}(x)\left(\mathcal{D}^{-1}\right)_{(x, y)} \eta(y)
$$

Inserting (35) into (37) we obtain

$$
\int_{x} \int_{y} \bar{\eta}_{v}^{+}(x) \frac{1+\psi}{2} e^{i M v \cdot x}\left(\mathcal{D}^{-1}\right)_{(x, y)} e^{-i M v \cdot y} \frac{1+\psi}{2} \eta_{v}^{+}(y)
$$


We are interested in computing the inverse of the operator $\mathcal{D}$ as a perturbation expansion in $1 / M$. We first notice that

$$
\mathcal{D}_{v}:=e^{i M v \cdot x} \mathcal{D} e^{-i M v \cdot x}=i \not D-2 M P_{-}+\Gamma \alpha_{\Gamma},
$$

where $P_{ \pm}$denote the following diagonal matrices of the velocity projection operators

$$
P_{ \pm}=\left(\begin{array}{cc}
\left(1 \pm \psi_{c}\right) / 2 & 0 \\
0 & \left(1 \pm \psi_{b}\right) / 2
\end{array}\right)
$$

and $\alpha_{\Gamma}$ is a matrix whose elements are given by

$$
\alpha_{\Gamma}^{i j}=e^{i m_{i} v_{i} \cdot x} A_{\Gamma}^{i j} e^{-i m_{j} v_{j} \cdot x} .
$$

$i, j$ take the values $c$ and $b$ correspondingly. $\alpha_{\Gamma}^{i j}$ must be considered a slowly varying source as well. The reason for this is that $A_{\Gamma}^{i j}$ must carry a momentum such that changes $m_{j} v_{j}$ into $m_{i} v_{i}$ up to small momentum fluctuations [6].

In the expression (39) the mass term contains zero modes, since it is multiplied by the projection operator. In the presence of zero modes the perturbation expansion around $\frac{1}{M}$ is not valid. In order to avoid this problem, we first decompose the operator $\mathcal{D}_{v}$ into the components $P_{ \pm}$as follows

$$
\mathcal{D}_{v}=\left(\begin{array}{cc}
i v \cdot D P_{+}+P_{+} \Gamma \alpha_{\Gamma} P_{+} & P_{+}\left(i \not D+\Gamma \alpha_{\Gamma}\right) P_{-} \\
P_{-}\left(i \not D+\Gamma \alpha_{\Gamma}\right) P_{+} & (-i v \cdot D-2 M) P_{-}+P_{-} \Gamma \alpha_{\Gamma} P_{-}
\end{array}\right)
$$

where all the operators are matrices in the flavor space. Again, at leading order, when $M \rightarrow \infty$, this operator is not invertible. We must, therefore, first invert the operator and then perform the $\frac{1}{M}$ expansion.

Next, we observe that the inverse $\left(\mathcal{D}_{v}\right)^{-1}$ can be written as

$$
\left(\mathcal{D}_{v}\right)^{-1}=\left(\begin{array}{cc}
1 & -R^{\prime} \\
0 & 1
\end{array}\right)\left(\begin{array}{cc}
A^{-1} & 0 \\
0 & B^{-1}
\end{array}\right)\left(\begin{array}{cc}
1 & 0 \\
-R & 1
\end{array}\right)=\left(\begin{array}{cc}
A^{-1}+R^{\prime} B^{-1} R & -R^{\prime} B^{-1} \\
-B^{-1} R & B^{-1}
\end{array}\right)
$$

where $A, B, R$ and $R^{\prime}$ are given by

$$
\begin{aligned}
A & =i v \cdot D P_{+}+P_{+} \Gamma \alpha_{\Gamma} P_{+} \\
B & =(-i v \cdot D-2 M) P_{-}+P_{-} \Gamma \alpha_{\Gamma} P_{-}-P_{-}\left(i \not D+\Gamma \alpha_{\Gamma}\right) \frac{1}{i v \cdot D+P_{+} \Gamma \alpha_{\Gamma} P_{+}} P_{+}\left(i \not D+\Gamma \alpha_{\Gamma}\right) P_{-} \\
R & =P_{-}\left(i \not D+\Gamma \alpha_{\Gamma}\right) \frac{1}{i v \cdot D+P_{+} \Gamma \alpha_{\Gamma} P_{+}} P_{+} \\
R^{\prime} & =P_{+} \frac{1}{i v \cdot D+P_{+} \Gamma \alpha_{\Gamma} P_{+}}\left(i \not D+\Gamma \alpha_{\Gamma}\right) P_{-}
\end{aligned}
$$


and the inverse, now, is defined in the space of projections, that is $A A^{-1}=P_{+}, B B^{-1}=$ $P_{-}$.

Then, we compute the inverse elements using perturbation theory and obtain the following result for the effective action at subleading order in $\frac{1}{M}$

$$
\begin{gathered}
\int_{x} \int_{y} \bar{\eta}(x)\left(\mathcal{D}^{-1}\right)_{(x, y)} \eta(y)=\int_{x} \int_{y} \bar{\eta}_{v}^{+}(x)\left\{P_{+} \frac{1}{i v \cdot D+P_{+} \Gamma \alpha_{\Gamma} P_{+}} P_{+}\right. \\
\left.-P_{+} \frac{1}{i v \cdot D+P_{+} \Gamma \alpha_{\Gamma} P_{+}}\left(i \not D+\Gamma \alpha_{\Gamma}\right) \frac{1}{2 M} P_{-}\left(i \not D+\Gamma \alpha_{\Gamma}\right) \frac{1}{i v \cdot D+P_{+} \Gamma \alpha_{\Gamma} P_{+}} P_{+}\right\}_{(x, y)} \eta_{v}^{+}(y) \\
\text { +higher order terms. }
\end{gathered}
$$

The last expression gives the generating functional for heavy quarks of velocity almost $v$. In order to describe a system with additional quarks of different velocity, for instance $v^{\prime}$, one needs to add to $\eta_{v}$ in (35) an analogous component $\eta_{v^{\prime}}$. Then apart from the contribution (44) and an analogous contribution for $v^{\prime}$ one gets terms mixing $v$ and $v^{\prime}$. These terms consist of slowly varying functions multiplying the oscillating exponentials of the kind $\exp \left\{-i M\left(v-v^{\prime}\right) \cdot x\right\}$. We prove below that these terms can be put to zero at any finite order in $1 / M$, as far as $M\left(1-v \cdot v^{\prime}\right) \gg \Lambda_{Q C D}$.

The terms in question enter in the effective action as follows

$$
\int d x e^{-i M\left(v-v^{\prime}\right) \cdot x} f(x)
$$

where $f(x)$ is a slowly varying function. Equivalently (45) can be written as

$\int_{x} \frac{2}{-i M\left(v-v^{\prime}\right)^{2}}\left(v_{\mu}-v_{\mu}^{\prime}\right) \partial^{\mu} e^{-i M\left(v-v^{\prime}\right) \cdot x} f(x)=\frac{1}{-i M\left(1-v \cdot v^{\prime}\right)} \int_{x} e^{-i M\left(v-v^{\prime}\right) \cdot x}\left(v_{\mu}-v_{\mu}^{\prime}\right) \partial^{\mu} f(x)$.

Since $f(x)$ varies slowly by iterating the process these terms can be set to zero to any finite order in $\frac{1}{M}$.

Our expression for the generating functional (44) is non-local, since it contains the propagator in the denominator. It can be shown, however, that it can be derived from a local Quantum Field Theory. Namely, the exponential of the right hand side of (44) can be written as

$$
\frac{1}{\operatorname{det}\left(\mathcal{D}_{v}\right)} \int d \bar{h}_{v}^{+} d h_{v}^{+} e^{i \int_{x}\left(\bar{h}_{v}^{+} \mathcal{D}_{v} h_{v}^{+}+\bar{\eta}_{v}^{+} h_{v}^{+}+\bar{h}_{v}^{+} \eta_{v}^{+}\right)}
$$


where $\mathcal{D}_{v}$ is given by the following local expansion

$$
\mathcal{D}_{v}=i v \cdot D+P_{+} \Gamma \alpha_{\Gamma} P_{+}+\frac{1}{2 M}\left(i \not D+\Gamma \alpha_{\Gamma}\right) P_{-}\left(i \not D+\Gamma \alpha_{\Gamma}\right)+\text { higher order terms }
$$

Then, using (32) and (47) we obtain

$$
Z\left(\bar{\eta}, \eta ; A_{\Gamma}\right)=\frac{\operatorname{det} \mathcal{D}}{\operatorname{det} \mathcal{D}_{v}} \int d \bar{h}_{v}^{+} d h_{v}^{+} e^{i \int_{x}\left(\bar{h}_{v}^{+} \mathcal{D}_{v} h_{v}^{+}+\bar{\eta}_{v}^{+} h_{v}^{+}+\bar{h}_{v}^{+} \eta_{v}^{+}\right)},
$$

with $\mathcal{D}_{v}$ given as in (48). In our last expression the exponent at leading order coincides with the action $(3)$ and hence $h_{v}^{+}$can be identified with the heavy quark fields.

Let us ignore the determinants for a moment. By putting the sources equal to the gluon field in the last exponent our local Quantum Field Theory coincides with that given in ref. [6]. In this respect our result is equivalent with the result obtained by Mannel et al. as far as the above mentioned determinants can be dropped. Furthermore, by taking functional derivatives of our expression for the generating functional (49) with respect to $A_{\Gamma}^{i j}$ we generate the realization of all currents in the HQET. Our results for the currents can be shown to coincide with previous results at $O(1 / M)[6]$, again if the determinants can be dropped.

The determinants are the only ill-defined quantities in our generating functional (49). Keeping them explicit in our expressions amounts to keeping all the contributions leading to the chiral anomaly. In fact $\operatorname{det}(\mathcal{D})$ is known to give rise to the chiral anomaly [13]. On the other hand $\operatorname{det}\left(\mathcal{D}_{v}\right)$ can be defined in a chiral invariant way. This was shown in the formulas (29) to (31) at leading order. In fact the proof holds at any order in $1 / M$, since it is only based on the transformation properties of $\mathcal{D}_{v}$ and on the existence of the $\psi^{\prime}$ with ${\psi^{\prime}}^{2}=1$. This result is in agreement with ref. [6] where $\operatorname{det}\left(\mathcal{D}_{v}\right)$ was shown to be a constant by a different argument. Therefore, the theory described by (49) and (48) fulfills trivially the 't Hooft anomaly matching condition. The locality of the theory is assured, since $\operatorname{det} \mathcal{D}$ admits a local expansion in $1 / M$ (we consider the Wess-Zumino term as local) the leading terms of which have been given in ref. [13]. (See [14] for the case of Majorana masses). Some next-to-leading contributions have been recently calculated in [15]. We shall not display them explicitly here.

The extra non-trivial contribution $\operatorname{det} \mathcal{D}$, present in our approach, has not played any role in the phenomenological applications of the HQET considered so far, since its contribution to the currents is zero in the first order in $1 / M$. Nevertheless, it gives extra 
contributions to the currents in the HQET which in principle can contribute in some higher order processes.

\section{Conclusions}

In conclusion, we have examined the anomalies in the effective theory of heavy quarks. We have shown that neither the spin nor the flavor symmetries contain an anomaly in Georgi's lowest order approximation of the effective theory for the heavy quarks. Moreover, we have pointed out the existence of an extra symmetry in this theory and have shown that this is also free of anomaly.

In the second part of this work we investigated the question of the chiral anomaly of the fundamental (QCD) theory. For this purpose we have enlarged the original theory in such a way as to keep the chiral symmetry explicit. We have, then, shown that the heavy quark effective theory, obtained from this theory by just field redefinition, is anomaly free.

Then, in order to keep track of any contribution due to the anomaly, we have given an alternative derivation of the effective theory of heavy quarks. In particular, using the generating functional method we are able to compute in a sytematic way higher order corrections in the $1 / m$ expansion. By explicitly keeping the determinant in this pathintegral formalism, we account for all possible corrections due to the anomaly in matrix elements. The processes, however, in which the determinant would be relevant typically correspond in QCD loop corrections to second order electroweek processes and hence they are very suppressed. This is probably the reason why our extra determinant contribution has been unnoticed by previous authors.

Finally, we emphasize that our results obtained for the effective currents and the local Quantum Field Theory coincide with previously obtained results as far as the contributions from the determinant can be ignored.

\section{Acknowledgements}

We thank D. Espriu, A. Müller, A. P. Polychronakos, E. de Rafael and A. Slavnov for discussions. We are specially indebted to J. Taron for introducing us to the HQET, many discussions and a careful reading of the manuscript. 


\section{References}

[1] N. Isgur and M.B. Wise, Phys. Lett. B232 (1989) 113; and Phys. Lett. B237 (1990) 527.

[2] E. Eichen and B. Hill, Phys. Lett. B234 (1990) 511; M.E. Luke, Phys. Lett. B252 (1990) 447; A.F. Falk and B. Grinstein, Phys. Lett. B247 (1990) 406; A.F. Falk and B. Grinstein Phys. Lett. B249 (1990) 314; A.F. Falk, B. Grinstein and M.E. Luke Nucl. Phys. B357 (1991) 185; A. F. Falk, H. Georgi and B. Grinstein, Nucl. Phys. B343 (1990) 1; H. Georgi Nucl. Phys. B348 (1991) 293; H. Georgi, B. Grinstein and M.B. Wise Phys. Lett. B252 (1990) 456; T. Mannel, W. Roberts and Z. Ryzak, Phys. Lett. B271 (1991) 421 and Phys. Rev. D45 (1992) 875.

[3] B. Grinstein, preprint HUTP-91/A028 (1991) and HUTP-91/A040, SSCL-Preprint-17 (1991).

[4] W.E. Caswell and G.P. Lepage Phys. Lett. B167 (1986) 437; G.P. Lepage and B.A. Thacker, Nucl. Phys. B4 (Proc. Suppl.) (1988) 199.

[5] H. Georgi Phys. Lett. B240 (1990) 447.

[6] T. Mannel, W. Roberts and Z. Ryzak, Nucl. Phys. B363, (1991) 19.

[7] S.L. Adler and W.A. Bardeen, Phys. Rev. 182 (1969) 1517.

[8] C. Bouchiat, J. Iliopoulos and Ph. Meyer,Phys. Lett. B38 (1972) 519; D.J. Gross and R. Jackiw, Phys. Rev. D6 (1972) 477.

[9] G. 't Hooft, in "Recent Developments in Gauge Theories”, G. 't Hooft et al. (Eds.), Plenum Press, New York, 1980.

[10] S. Coleman and B. Grossman, Nucl. Phys. B203 (1982) 205.

[11] J. Gasser and H. Leutwyler, Ann. Phys. (N.Y.) 158 (1984) 142.

[12] L. Alvarez Gaumé and P. Ginsparg, Nucl. Phys. B243 (1984) 449.

[13] R.D. Ball, Phys. Rep. 182 (1989) 1, and ref. therein; E. D'Hoker and E. Fahri, Nucl. Phys. B248 (1984) 77; R.D. Ball and H. Osborn, Nucl. Phys. B263 (1986) 245.

[14] J.L. Goity and J. Soto, Phys. Lett. B233 (1989) 400.

[15] J. Bijnens, Nucl. Phys. B367 (1991) 709. 